\title{
NEW TOOL TO HELP DECISION MAKING IN CIVIL ENGINEERING
}

\author{
Enrique CASTILLO ${ }^{a}$, Jose Antonio LOZANO-GALANT ${ }^{\mathrm{b}}$, Maria NOGAL ${ }^{\mathrm{c}}$, Jose TURMO ${ }^{\mathrm{d}}$ \\ ${ }^{a}$ Department of Applied Mathematics and Computational Sciences, University of Cantabria, 39005 Santander, Spain \\ ${ }^{b}$ Department of Civil Engineering, University of Castilla-La Mancha, 13071 Ciudad Real, Spain \\ ${ }^{c}$ Departament of Civil, Structural \& Environmental Engineering, Trinity College Dublin, Dublin, Ireland \\ ${ }^{d}$ Department of Construction Engineering, Universitat Politècnica de Catalunya BarcelonaTech, Spain
}

Received 6 August 2012; accepted 08 Jan 2013

\begin{abstract}
Structural collapses have indirectly produced important progress in science. The last lesson learnt from structural collapses reveals the important role of polyrational systems of equations in the Civil Engineering field, as all problems in Physics and Engineering involving scale variables lead to systems of these equations. Since no efficient methods are known to solve this type of equations, a powerful and efficient method to multivariate polyrational equations using observability techniques is presented in this paper. It is pointed out that this method can be applied to solve different Civil Engineering problems. The information obtained by this tool can be used to assist decision making and risk management processes during maintenance and service life. As an example of its use, a structural damage detection problem is solved.
\end{abstract}

Keywords: polyrational systems of equations, observability, decision making process, structural system identification.

\section{Introduction}

The history of humanity is closely linked to structural damage and to the loss of the load-carrying capacity of their components that may produce collapse. From ancient times, the collapse of an almost endless number of constructions, such as cathedrals, bridges or dams, have caused important social and environmental impacts, with associated economic costs, especially in terms of human lives. Due to its importance, the collapse of a structure nowadays receives significant attention from researchers.

Structural damages can also be interpreted as an indirect mean for to developing science. The collapse of bridges due to resonance produced by military steps led to new precautions in bridge design or when dealing with dynamic loads. Failures of the Dee Bridge, in Great Britain (1847), or the Tay Bridge in Scotland (1879), led to improving the knowledge of steel (Akeeson 2008). From ancient times, failures have permitted us to identify new phenomena, such as the time-dependent behaviour of concrete material discovered after checking the deflections of Le Veurdre Bridge (1910), which produced the first documented prestressing application by Freyssinet, the aeroelastic flutter phenomenon discovered after the collapse of the Tacoma Narrows Bridge (1940) by the team led by Bleich et al. 1950, and the synchronous lateral excitation phenomenon in the Millenium Bridge in London (2000). Unfortunately, as in any other problem in science, failure of a structure due to a certain phenomenon cannot be avoided until it is discovered, studied and wellunderstood. Only when this process has been completed can theoretical models which simulate the behaviour of the real phenomenon be constructed and the simulation of the real structure behaviour can be achieved with high precision.

Since structures deteriorate with time, it is important to differentiate between models used in design (where material characteristics are assumed) from those utilized during service life, when material properties could have changed. According to a Federal Highway Administration report, in the USA alone, more than 160,000 bridges were structurally or functionally deficient in 2004. During service life, inspections are often required to guarantee safety requirements. However, mathematical models should also be adapted to incorporate changes in the material and physical properties, but it is not always the case. An example of this is found in the I-35W Mississippi River Bridge (USA) presented in Figure 1a and 1b. Despite the fact that this bridge was annually inspected, its collapse occurred in 2007, killing thirteen and injuring one hundred and fortyfive people. Unfortunately, the inspections were not used to know the real stress state of the structure or its actual material characteristics, which could have been done by updating the structural model. This example proves that even regular inspections cannot guarantee structural

Corresponding author: Jose Antonio Lozano-Galant

E-mail: joseantonio.lozano@uclm.es 
(a)

(b)
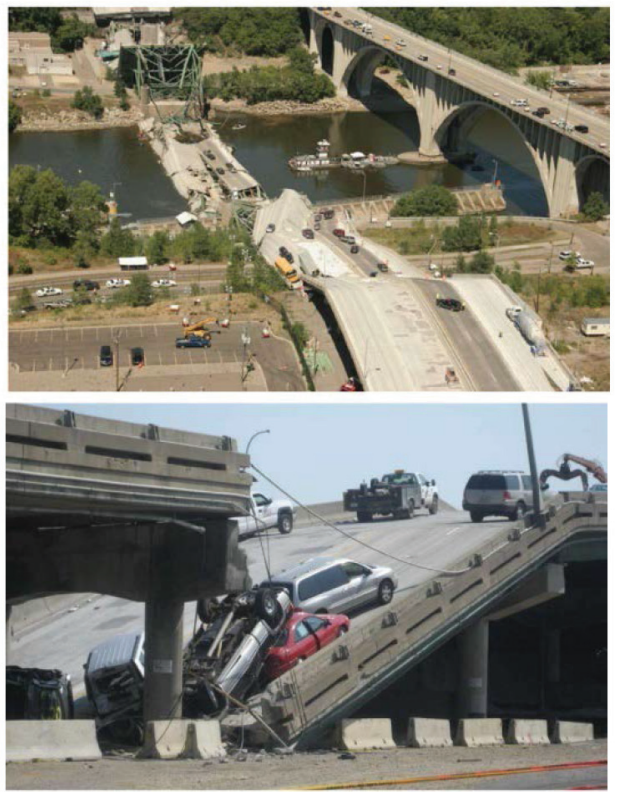

Fig. 1. Collapse of the I-35W Mississippi River Bridge, USA (courtesy of Federal Highway Administration and Context Sensitive Solutions and Creative Commons ${ }^{1}$ )

safety. To avoid such failures, the information obtained by inspection programs should be used to calibrate theoretical models, to reproduce the actual structural behaviour and to locate structural damages more accurately. This information must be linked with decision making (Simanaviciene et al. 2012; Liaudanskiene et al. 2012) and risk management processes (Kim 2010; Paslawski 2011; Turskis et al. 2012) to assure structural safety and functionality while the structure is in service.

In the last few decades the development of the Health Monitoring field enabled the identification of structural parameters of the actual structure from information obtained on site. This technique is known as Structural System Identification (SSI), a complex process to determine the actual parameters of a structural system based on a subset of measured inputs and outputs. One of the most important applications of the SSI is damage detection (Garstecki et al. 2004; Yonggang et al. 2008).

Damage can be detected by non-destructive tests that include the response of the structure to a certain excitation. According to the excitation, these experiments can be classified as dynamic or static according to whether or not they engage inertial effects. Most of the damage detection methods proposed in the existing literature are based on dynamic excitement (Witzany, Cejka 2007; Zwolsky, Bien 2011).

A wide range of damage detection methods has been presented in the existing literature. These can be classified as parametric, in which the parameters of an actual system model are directly used to represent the physical properties of the structure, and non-parametric methods, in which the input-output relation is characterized and determined by a set of equations that may not

\footnotetext{
${ }^{1}$ http://www.flickr.com/photos/kj415/1056861837/
}

have any explicit physical meaning, which is their main drawback. During the last decades, computer development has enabled increasing popularity in non-parametric methods such as Neural Networks (Talebinejad et al. 2011) or Genetic Algorithms (Marano et al. 2011), as complex structural systems can be identified, whereas parametric methods have mainly been applied to very simple models, due to the complexity of the mathematical relations among the variables.

After this introduction, it becomes clear that structural failures foster new mathematical models and techniques of solution. In this paper we show that the history repeats itself. When analysing the real state of a structure, we showed that systems of polyrational equations (equations involving monomial ratios of variables such as, $X^{2} Y$ or $3 X^{5} Y^{1 / 3}$ being $X$ and $Y$ the variables of the system) arise in structural analysis. Later we realized that this new mathematical problem appears in all fields of science and engineering where scale variables are involved. Since no satisfactory methods to solve these systems of equations exist, a static excitement based, analytical, parametric and efficient method that avoids resorting to numerical approximations is presented. This new parametric tool can help the decision making process to support operational and maintenance decisions on site.

Structure of the paper is as follows. In Section 1, the importance of the polyrational systems of equations in all fields in science and engineering is presented. In this section a field where polyrational systems of equations appear (SSI) is described in depth. In Section 2, the observability techniques are applied to solve polyrational systems of equations. In Section 3 the application of the proposed methodology to the decision making in a real bridge is presented. Finally, in the last section, some conclusions are drawn.

\section{Polyrational systems of equations}

It is very important to mention that the fact that we get polyrational equations when writing a model for physical and engineering problems is not a coincidence, but the result of physical formulas to be independent on the units of measure (Aczél 1966a, 1967; Castillo, Ruiz-Cobo 1999; Castillo et al. 2005). Thus, we should get the same type of polyrational equations in any problem arising from physics or engineering. More precisely, Aczél 1966b demonstrated that the general form of dependent real-valued variables with ratio scale non-constant and continuous-at-a-point when all fundamental or independent variables have ratio scale, are functions of the form:

$$
u\left(\mathrm{x}_{1}, \mathrm{x}_{2}, \ldots, \mathrm{x}_{n}\right)=a \prod_{i=1}^{n} x_{i}^{c_{i}}=a \frac{\prod_{c_{i}>1}^{n} x_{i}^{c_{i}}}{\prod_{c_{i}<1}^{n} x_{i}^{c_{i}}},
$$

where: $a$ is a constant; $x_{1}, x_{2}, \ldots, x_{n}$ are the variables involved in the equation; and $c_{1}, c_{2}, \ldots, c_{n}$ are negative or 
positive real constants. Some examples of such equation in physics are the kinetic energy formula or the universal gravitation law:

$$
E_{k}=\frac{m v^{2}}{2}, \quad F=G=\frac{m_{1} m_{2}}{r^{2}} .
$$

This means that no other formulas are possible from the point of view of unit consistency. A detailed analysis of this problem has been carried in Castillo and Ruiz-Cobo (1999), Castillo et al. (2005, 2012).

This important property makes clear that polyrational equations play a very relevant role in physics and engineering fields. As some examples, we first can mention the cases of electrical and water power distribution.

In these two cases, knowing the state of the distribution conduction or lines is crucial. For example, one of the main problems when the New York blackout occurred was the inability to pinpoint the exact site of the failure (Firestone, Pérez-Peña 2003). This led to an unusual flurry of accusations on both sides of the international border. Canadians initially said that failure originated due to a fire in a power plant near Niagara Falls on the American side, possibly caused by a lightning strike. This hypothesis was shortly replaced by saying that the power failure originated at a nuclear power plant in Pennsylvania. American officials denied both charges and indicated that the event began somewhere west of the Ontario power system, taking out electric power in Ontario and parts of Michigan and Ohio that produced the New York blackout. Later, New York State officials said the event may have begun with a power surge at the Perry nuclear power plant near Cleveland (Firestone, Pérez-Peña 2003).

As a consequence, several investments of billions of dollars in new wires, towers and transformers were suggested to prevent such power failures in the future. However, much cheaper and more efficient methods are available, based on maintenance and control policies which permit identifying the exact location of critical or damaged elements and thus replacing not all but only selected critical elements, with considerable savings in money and materials. This is where the calibration and identification of the state of the system arises as a powerful and useful technique.

We also find polyrational systems of equations in the water supply problem, where the node flow balance equations, pipe height losses written in terms of the water velocities and the roughness and diameter of the pipes, and the total node heights written in terms of the geometric, piezometric and cinetic heights lead to a system of polyrational equations. A complex problem to be solved in water supply systems is the location of obstructed pipes which substantially degrades the efficiency of the network. It is clear that the methods proposed in this paper permit us to locate the sources of failures by adequately measuring pressures and speeds at adequately selected locations.
Another field of application is high-speed train safety. The accident suffered by the InterCityExpress train set 51, travelling on the Munich to Hamburg route, could have been avoided if adequate maintenance policies were applied, permitting us to detect the location of damaged elements. The result of the accident can be thus summarized as: 101 fatalities, 88 severe injuries and 106 minor/ no injuries.

According to some information, during the week prior to the Eschede disaster, three separate automated checks indicated that a wheel was defective. Investigators discovered from a maintenance report generated by the train's on-board computer that two months prior to the Eschede disaster conductors and other train staff filed eight separate complaints about the noises and vibrations generated from the bogie with the defective wheel but the company did not replace the wheel. The proposed technique of polyrational systems can be successfully applied to prevent failures or damage to train elements which can be identified by designing an appropriate maintenance protocol.

Hence, it is clear that polyrational equations play a very relevant role in physics and engineering fields such as: 1) the water supply problem (Castillo et al. 2012); 2) the electrical power distribution problem; 3 ) the highspeed train safety problem; and 4) the Structural System Identification problem.

The application of the polyrational systems of equations appearing in the Structural System Identification is presented in this paper as an example of the application of such technique.

\subsection{Polyrational system of equations in structural system identification.}

In structural analysis, statement of the equilibrium conditions leads together with strength of materials theory to the following system of equations:

$$
[K] \times\{\delta\}=\{f\},
$$

in which $[K]$ is the stiffness matrix of the structure that depends on material and geometric properties of the structural elements; $\{\delta\}$ is a vector of node displacements (in 2D horizontal and vertical deflections $u_{i}, v_{i}$ and rotations $w_{i}$ in each node); and $\{f\}$ is a vector of node forces (in 2D horizontal and vertical forces $H_{i}, V_{i}$ and moments $M_{i}$ in each node).

As an illustrative example, a simply supported beam is presented in Figure 2a. In Figure 2b, the different terms of Eqn (1) for 2D analysis are shown, including the geometrical and mechanical properties of the beam elements of the structure, such as length, $L$, Young's modulus, $E$, area, $A$, and inertia, $I$.

The stiffness method assumes that the stiffness matrix $K$ is known, and since the stiffness matrix is singular, the system of equations has infinite solutions.

Then, the engineer adds the boundary conditions of the structure and a new system with a unique solution is obtained. The number of boundary conditions depends 


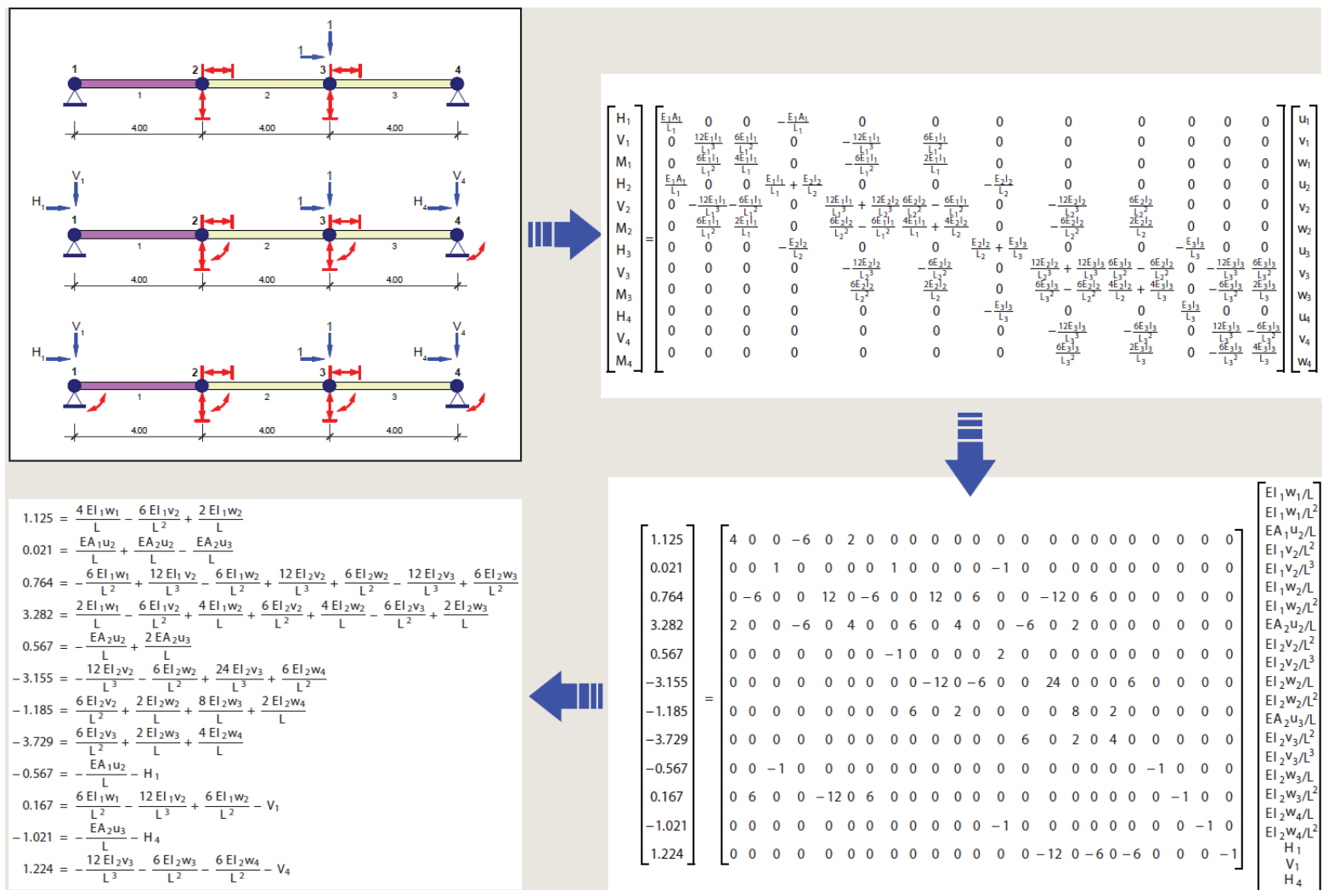

Fig. 2. Example of a simple beam showing the initially measured displacements and forces and the resulting observed extra displacements and forces (reactions) after recursive steps 1 and 2 (a); the resulting stiffness matrix $[K]$ and force $\{f\}$ and displacement $\{\delta\}$ vectors (b); the modified stiffness matrix $K^{*}$ and the monomial products located in the column matrix of the left hand side (c); the system of multivariate polyrational (polynomial) function, which in this case degenerates to a multivariate polynomial system of equations (d)

on the structural redundancy of the structure and must avoid the presence of global or partial mechanisms. For a 2D structure, a minimum of three boundary conditions (e.g. in structure of Figure $2 \mathrm{a} u_{1}=0, v_{1}=0$ and $v_{4}=0$ ) is required. In this case, the system of equations is linear and easy to solve.

The stiffness method is a direct method, as the mechanical and geometrical properties of the structure are known. However, due to uncertainties in the materials, construction procedures, stress state, degradation or damage, this is not always the case. Thus, the inverse problem is introduced in the SSI where $[K]$ is at least partially unknown. In summary, while direct methods solve systems of linear equations to determine reactions and node displacements in terms of forces, boundary conditions and material and geometric properties, inverse methods solve polyrational systems of equations to determine material and geometric properties in terms of boundary conditions and displacements (Castillo et al. 2012). This enables us to determine material parameters in the stiffness matrix (output), based on producing changes in the vector of forces and measuring the vector of displacements (input) in order to make better predictions. Even more, it allows us to locate structural damage and assess the corresponding magnitude. Unfortunately, the resulting system of equations in this case is non-linear. In this ar- ticle we present mathematical models that allow us to solve this non-linear system, that is to say, the inverse problem in very general cases.

If we assume that the coefficients in matrix $K$ are no longer constant and involve some unknown variables we face the inverse problem (Fig. 2b). Then, the unknown terms in the stiffness matrix can be moved to the right column vector so that we keep a constant coefficient matrix and we obtain a column vector of non-linear unknown variables (Fig. 2c). Note that: (a) terms of matrix $K$ involved unknown variables such as: $\frac{E A}{L}, \frac{E I}{L^{2}}$ or $\frac{E I}{L^{3}}$; (b) these unknown variables are non-linearly related and (c) unknown variables present in $K$ are also multiplying those present in $\delta$. This implies that our new unknown variables are not $E, A, I, L$ or $u_{i}$, but products/quotients of them, as $\frac{E A u_{i}}{L}, \frac{E I v_{i}}{L^{2}}$ or $\frac{E I v_{i}}{L^{3}}$. Thus, we obtain unknown variables that are quotients of multivariate mo-

nomials (very simple rational functions), which in some cases can degenerate to single monomials. Consequently, we obtain a system of polyrational equations, that is, linear combinations of elemental rational functions (Fig. 2d). Moreover, the linearized products or quotient variables outnumber the original ones (Fig. 2c). 


\section{Solving polyrational systems of equations}

Though there are very well-known and efficient methods for solving very large systems of linear equations, we have not found in the existing literature any works dealing with the problem of solving this type of multivariate polyrational equations. However, this is not the case for the particular case of polynomial equations. The field of multivariate polynomial equations has been studied in the existing literature. A good reference is Lazard (2009), in which a summary of the state of the art on polynomial equations is given. In this area, Gröbner bases, which are introduced by Buchberger $(1965,1970$, 1976), are the key tool. Important references in Gröbner bases are Buchberger (2001) and Faugère and Perret (2009), but there are many others. Unfortunately, the Gröbner bases techniques for solving polynomial equations are not very efficient and do not include the case of negative exponents in the factors (polyrational equations). Thus, new mathematical methods are required to solve not only multivariate polynomial equations, but the more general problem of multivariate polyrational equations.

Given that no practical solving methods are available to our knowledge for these equations, we propose the use of observability analysis to the SSI field.

\subsection{Observability}

In many areas of engineering the variables involved in a given problem are related by constraints that must be satisfied in order to make the solution of the problem physically valid. These constraints frequently lead to systems of equations that have an infinite number of solutions when they are initially stated (Castillo et al. 1999). Thus, the engineer must add new constraints (boundary conditions) for the problem to have a unique solution. However, it could happen that the system, which does not have a unique solution for all variables, it could have a unique solution for a proper subset of variables (Castillo et al. 2000, 2002). We say that a subset of variables is observable when the system of equations implies a unique solution for this subset, even though the remaining variables remain undetermined. This leads to the observability problem, which has a relevant role in many engineering problems. In particular, given a system of equations we can ask several questions that are relevant to SSI problems. Among them are the following:

1) What is the subset of variables with a unique solution?

2) What is the minimum subset of variables needed to uniquely obtain the values of another subset of variables?

3) What is the subset of variables that become observable given another subset of variables?

Castillo et al. (2007) provided a general method to solve the observability problem in the case of systems of linear equations or inequalities. However, since many problems in engineering are non-linear, this method cannot be directly used in many practical cases.

The application of observability techniques to solve polyrational system of equations in SSI is much more complex than to some previous applications (Lozano-Galant et al. 2012). Since the system of equations in terms of the initial variables is non-linear, these techniques are not directly applicable, but they can be applied to the system of equations in terms of products or quotients because they are linear. Once we have obtained the values of the elemental product or quotients, we can take logarithms to obtain new linear systems that allow us to obtain the values of some initial variables.

\subsection{Developed method}

Recently, the authors have developed a technique that promises to solve the polyrational equations arising in some engineering problems (Castillo et al. 2012). In particular, they show that it is very efficient in solving the problems arising from the structural field.

Figure 3 shows a flow chart of the proposed method. The main idea consists of finding the unique solutions of elemental rational ratios (products of single variables raised to positive or negative powers), which is denoted "learning products step" in Figure 3, and then learning the signs and the absolute values of the variables, solving the corresponding systems in terms of single variables by taking logarithms denoted "learning single variables step". A "simplification step", in which the actual knowledge of data or derived values of the variables is used to simplify the actual product variables, completes the three main steps of the algorithm.

Since the resulting observed variables in each iteration are not included in the observability analysis corresponding to that iteration, a recursive process has to be carried out where the observable information of preceding iterations is introduced as new input data. This process has to be repeated until no additional observable variables are obtained between two successive steps. The recursive process allows us to analytically solve the polyrational system of equations without the need to resorting to numerical approximations.

The proposed method can be formally explained as follows:

INPUT: The topology of the structure and the subset $\boldsymbol{M}$ of known mechanical properties and measured deflections.

OUTPUT: The resulting subset of observable variables $\boldsymbol{O}$ (subset of single and/or product variables) that can be written in terms of the subset $\boldsymbol{M}$ of measured variables.

Step 1: Build the stiffness matrix of the structure $K$ and its modified version $K^{*}$. Based on the topology of the structure the stiffness matrix $K$ is built. Modify the stiffness matrix $K$ to $K^{*}$ by separating the terms made of summands such that each summand correspond to a single column. 


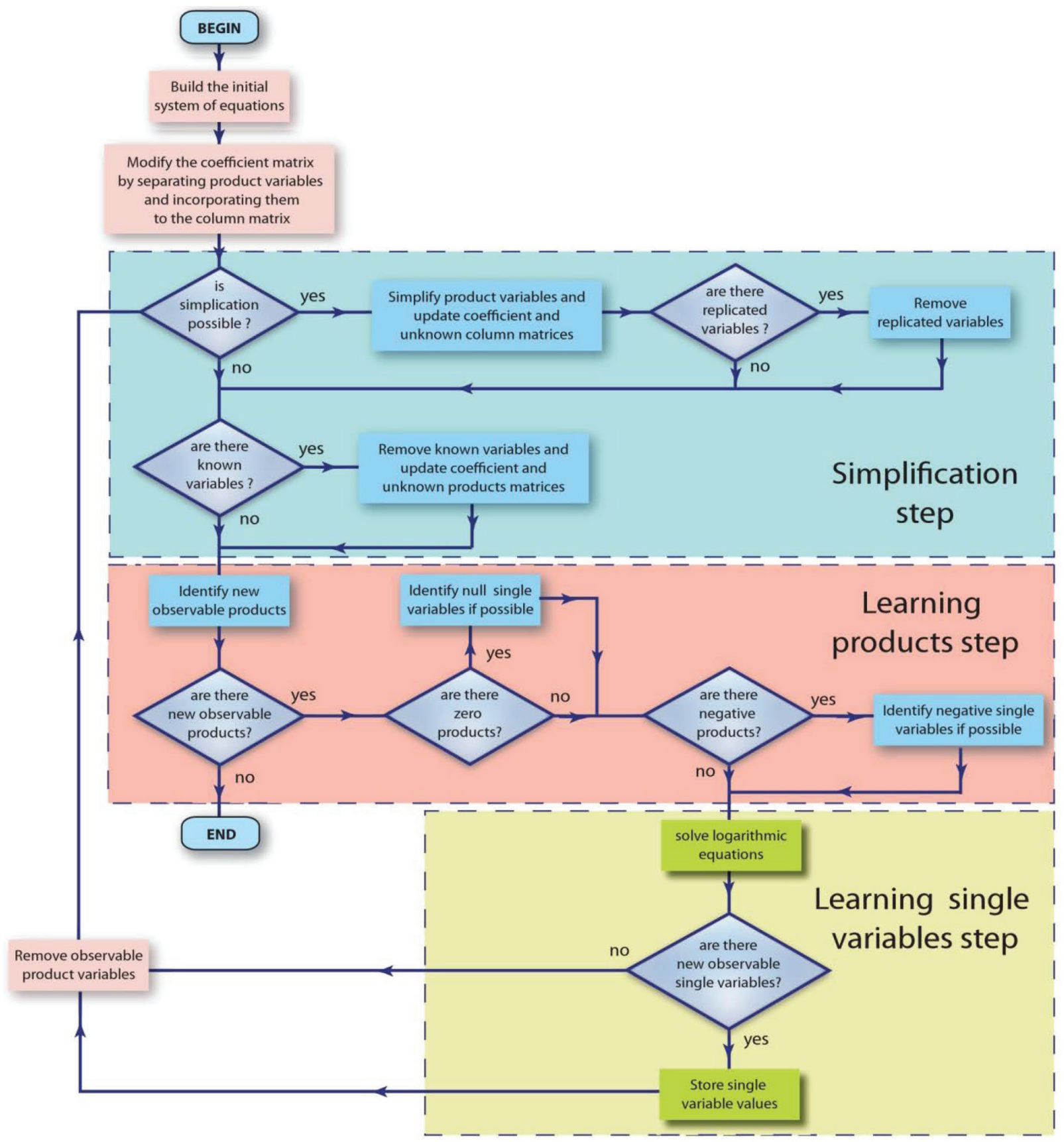

Fig. 3. Flow chart of the proposed method for solving the system of multivariate polyrational equations, showing the simplification step and the learning steps 1 and 2

Step 2: Generate the list of product variables. Identify the set of all unknown variables in $K$ and multiply it by $\delta$. A new vector $\delta^{*}$ of different product variables (such as $E A u, E I v$ or $E I w$ ) is obtained. After this step, matrix transforms to a constant and known matrix $K^{*}$. Matrix $K^{*}$ can be rearranged in order that $\delta^{*}$ contains only monomial terms.

Step 3: Impose boundary conditions and known loads. Replace the displacements and known loads associated with the boundary conditions by the corresponding values.
Step 4: Remove the measured variables and update. Multiply the columns of $K^{*}$ associated with measured variables by their corresponding values and remove the associated factors from the column matrix $\delta^{*}$.

Step 5: Eliminate duplicated variables and remove the null columns. Identify duplicated variables in $\delta^{*}$, compact matrix $K^{*}$ by adding columns of duplicated variables and replace column by the resulting non-duplicated variable list.

Step 6: Reorder the system. Reorder the system such that the unknown variables $\{z\}$ are grouped together 
and the known values appear in the independent term column matrix $D$. The resulting system has associated a matrix $B$, that is, the system becomes:

$$
[B]\{z\}=\{D\} .
$$

Step 7: Obtain the null space of matrix $B,[V]$. In order to derive the observable variables the null space of matrix $B$ is obtained (see note).

Step 8: Identify the observable variables. Identify the null rows of the matrix with the generators of the null space. The associated product variables are the observable variables (they have a unique solution) whose values are obtained.

Step 9: Identify null single variables. If the unique values for the observable product variables are null, the associated null displacements ( $E, A$ and $I$ values cannot be null) are identified.

Step 10: Identify negative single variables. If the single values for the observable product variables are negative, the associated negative displacement ( $E, A$ and $I$ values cannot be negative) are identified.

Step 11: Solve logarithmic equations. By taking logarithms, build the linear system involving absolute values of observable product variables and obtain the corresponding null space.

Step 12: Identify observable single unknown variables. These variables correspond with null rows of the null space, as in Step 9. The absolute values of these variables are obtained and if their signs have been determined, the values become known.

Step 13: Recursive Process. Repeat the process from Step 4 to Step 13 again with the values of observable variables until no new observable variables are obtained. In such a case return the list of all observable variables $\boldsymbol{O}$ and the corresponding values.

Note: The general solution (the set of all solutions) of the system (4) has the structure (Castillo et al. 2000, 2002):

$$
z=z_{0}+[V] \times \rho,
$$

where: $z, z_{0}$ and $\rho$ are column matrices; and $[V]$ is a rectangular matrix of arbitrary real values, that contains the null space generators. The solution of a given $z$ is unique if, and only if, the corresponding row in matrix $V$ is null. In such a case, the corresponding unique value is in the corresponding element of $z_{0}$.

It is important to note that in some particular cases (the field of structures presents some examples) these multivariate polyrational functions become multivariate polynomial functions, but in general this is not the case. In this case, we obtain a multivariate polynomial system of equations because we have assumed that the lengths of the elements are known, otherwise we obtained a rational monomial function.

In the following section the application of the proposed methodology to damage detection is presented by its application in an actual structure. Then, some insights to help the decision making process when damages are detected are provided.

\section{Application of the method}

To illustrate the proposed methodology, an example where the technique is used for damage quantification, the Koror-Babelthuap Bridge in the Republic of Palau (Fig. 4a) is described. This structure was a prestressed
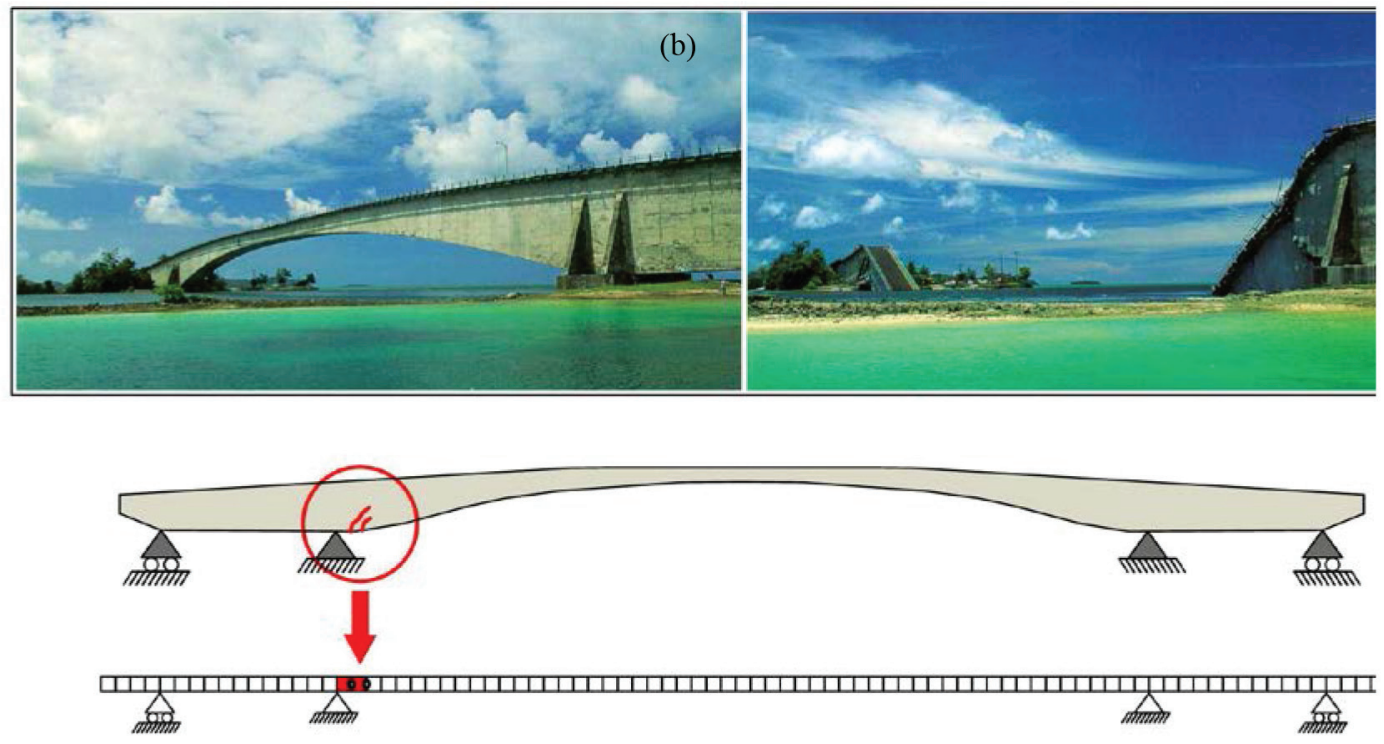

Fig. 4. Koror-Babelthuap bridge in Republic of Palau: (a) Actual structure (Courtesy of Bill Perryclear and Chris Burgoyne); (b) Collapsed structure (Courtesy of Bill Perryclear and Chris Burgoyne); (c) Detected Cracks (Burgoyne, Scantlebury 2006); and (d) Finite Element Model 
concrete bridge with a total length of $385 \mathrm{~m}$, which collapsed in 1996 (Fig. 4b), killing two people and injuring other four.

The actual characteristics of the built structure were unknown due to uncertainties in the materials, construction procedures and stress state. As the bridge was made of concrete, flexural and axial stiffness (EI, EA) might have been influenced by the stress state of the structure. The damage scenario could have been reproduced, according to the actual damage of the structure reported in Burgoyne and Scantlebury (2006). In this study, structural cracks, among others, were detected in the proximities of the left inner support, as presented in Figure 4c. A simplified Finite Element Model including 86 beam elements could have been used to quantify the damage on the structure. The characteristics of all beam elements except those located in the proximities of the damage (in red in Fig. 4d) might have been assumed to be known. The damaged flexural stiffness could have been obtained, providing that enough vertical deflections were measured in the actual structure under a given load test, (e.g. with some heavy trucks loading the deck). Assuming that the characteristics of the undamaged sections were known, flexural stiffness of the damaged section could have been obtained by measuring vertical deflections in the actual structure at two points (highlighted with circles in Fig. 4d).

The proposed methodology is not only limited to damage quantification when the location of the damage is known, it can also be used to determine the location of the damage along with its intensity. However, more information is required to do so as the flexural stiffness of all the beam elements are assumed to be unknown. In this example, in order to locate the damage, vertical deflections at 169 points in the structure should have been measured on site for a given load test. The flexural stiffness of all the 86 beam elements modelling the structure could have been obtained after 29 recursive steps.

As the static vertical deflections in actual structures can be economically and accurately measured by topography, the proposed method is presented as a powerful alternative to other damage detection methods proposed in the literature.

Comparing the real stiffness with the theoretical one, a damage index for the different elements of the structure can be determined, along with their remaining strengths. This information can be included in decision making and risk management processes to prevent future failures. Once the threshold value is reached in any of the elements of the structure, action might be taken.

\section{Conclusions}

In this paper, we have shown that the search for a method to avoid structural collapse has led to the knowledge of the importance of multivariate polyrational equations and to the development of new mathematical algorithms and procedures to solve them. As polyrational systems arises in science and engineering fields whenever scale variables are involved, some practical applications of this method in several fields of engineering are pointed out. As an example, the proposed methodology has been applied for locating and quantifying damages in actual structures based on static monitoring information obtained during inspection programs. This new tool can be included in decision making and risk management processes to design appropriate maintenance protocols or to identify failure in a complex system. The fact that the method is parametric enables not to lose the physical meaning of the estimated variables.

\section{Acknowledgements}

The authors are indebted to the Secretary of State of the Spanish Ministry of Science and Innovation (Project TRA2010-17818, BIA2009-10483 and BIA2009-13056) and to the Junta de Comunidades de Castilla-La Mancha (Project PII2I09-0129-4085), for partial support of this work.

\section{References}

Aczél, J. 1966a. Lectures on functional equations and their applications. Mathematics in Science and Engineering. Academic Press. 509 p.

Aczél, J. 1966b. Lectures on functional equations and their applications. 19, Mathematics in Science and Engineering. Academic Press.

Aczél, J. 1967. A short course on functional equations based upon recent applications to the social and behavioral sciences. Reidel Publishing Company. 169 p.

Akeeson, B. 2008. Understanding bridge collapses. London: Taylor \& Francis Group. 276 p.

Bleich, F.; McCullouh, C. B.; Rosecrans, R.; Vincent, G. S. 1950. The mathematical theory of vibration in suspension bridges. US Government Printing Office, Washington. $429 \mathrm{p}$.

Buchberger, B. 1965. Ein Algorithmus zum Auffinden der Basiselemente des Testklassenringes nach einem nulldimensionalen Polynomideal. PhD thesis. University of Innsbruck, Austria (in German).

Buchberger, B. 1970. An algorithmical criterion for the solvability of algebraic systems of equations, Aequationes Mathematicae 4(3): 374-383 (in German). http://dx.doi.org/10.1007/BF01844169

Buchberger, B. 1976. Some properties of Gröbner bases for polynomial ideals, ACM SIGSAM Bulletin 10(4): 19-24. http://dx.doi.org/10.1145/1088222.1088224

Buchberger, B. 2001. Gröbner bases: a short introduction for systems theorists, in Proceedings of EUROCAST 2001 ( $8^{\text {th }}$ International Conference on Computer Aided Systems Theory-Formal Methods and Tools for Computer Science), 19-23 February 2001, Las Palmas de Gran Canaria, Spain.

Burgoyne, C.; Scantlebury, R. 2006. Why did the Palau Bridge collapse?, The Structural Engineer 84: 30-37.

Castillo, E.; Ruiz-Cobo, R. 1992. Functional equations in science and engineering. New York: Marcel Dekker. 327 p.

Castillo, E.; Cobo, A.; Jubete, F.; Pruneda, R. E. 1999. Orthogonal sets and polar methods in linear algebra: applications to matrix calculations, systems of equations and inequalities, and linear programming. New York: John Wiley \& Sons. 424 p. http://dx.doi.org/10.1002/9781118032893 
Castillo, E.; Cobo, A.; Jubete, F.; Pruneda, R. E.; Castillo, C. 2000. An orthogonally based pivoting transformation of matrices and some applications, SIAM Journal on Matrix Analysis and Applications 22(3): 666-68. http://dx.doi.org/10.1137/S0895479898349720

Castillo, E.; Jubete, F.; Pruneda, R. E.; Solares, C. 2002. Obtaining simultaneous solutions of linear subsystems of equations and inequalities, Linear Algebra and its Applications 346(1-3): 131-154. http://dx.doi.org/10.1016/S0024-3795(01)00500-6

Castillo, E.; Iglesias, A.; Ruiz-Cobo, R. 2005. Functional equations in applied sciences. Mathematics in Science and Engineering, Elsevier. 391 p.

Castillo, E.; Conejo, A.; Pruneda, R. E.; Solares. C. 2007. Observability in linear systems of equations and inequalities, Applications, Computers and Operation Research 34(6): 1708-1720. http://dx.doi.org/10.1016/j.cor.2005.05.035

Castillo, E.; Nogal, M.; Lozano-Galant, J. A.; Turmo, J. 2012. Solving some special cases of polynomial equations appearing frequently in physical and engineering problems, Applied Mathematical Modeling (under review).

Faugère, J. C.; Perret, L. 2009. An efficient algorithm for decomposing multivariate polynomials and its applications to cryptography, Journal of Symbolic Computation 44(12): 1676-1689. http://dx.doi.org/10.1016/j.jsc.2008.02.005

Firestone, D.; Pérez-Peña, R. 2003. The Blackout of 2003: The context reveals creaky system, experts believe, The New York Times. Aug. 15.

Garstecki, A.; Knitter-Piatkowska, A.; Pozorski, Z.; Ziopaja, K. 2004. Damage detection using parameter dependent dynamic experiments and wavelet transformation, Journal of Civil Engineering and Management 10(3): 191-197. http://dx.doi.org/10.1080/13923730.2004.9636306

Kim, S. G. 2010. Risk performance indexes and measurements systems for mega construction projects, Journal of Civil Engineering and Management 16(4): 586-594. http://dx.doi.org/10.3846/jcem.2010.65

Lazard, D. 2009. Thirty years of polynomial system solving and now?, Journal of Symbolic Computation 44(3): 222-231. http://dx.doi.org/10.1016/j.jsc.2008.03.004

Liaudanskiene, R.; Simanaviciene, R.; Ustinovichius, L. 2012. A model for solving structural, technological and safety problems, Journal of Civil Engineering and Management 18(1): $30-42$.

http://dx.doi.org/10.3846/13923730.2011.643551

Lozano-Galant, J. A.; Nogal, M.; Castillo, E.; Turmo, J. 2012. Application of observability techniques to structural system identification, Computer-Aided Civil and Infrastructure Engineering 28(6): 434-450.

http://dx.doi.org/10.1111/mice.12004

Marano, G. C.; Quaranta, G.; Monti, G. 2011. Modified genetic algorithm for the dynamic identification of structural systems using incomplete measurements, Computer-Aided Civil and Infrastructure Engineering 26: 92-110. http://dx.doi.org/10.1111/j.1467-8667.2010.00659.x

Paslawski, J. 2011. Flexibility as risk management option implemented in the bridge repair, The Baltic Journal of Road and Bridge Engineering 4(4): 258-266. http://dx.doi.org/10.3846/bjrbe.2011.33

Simanaviciene, R.; Liaudanskiene, R.; Ustinovichius, L. 2012. A new synthesis method of structural, technological and safety decisions (SyMAD-3), Journal of Civil Engineering and Management 18(2): 265-276. http://dx.doi.org/10.3846/13923730.2012.666504

Talebinejad, I.; Fischer, C.; Ansari, F. 2011. Numerical evaluation of vibration-based methods for damage assessment of cable-stayed bridges, Computer-Aided Civil and Infrastructure Engineering 26: 239-251.

http://dx.doi.org/10.1111/j.1467-8667.2010.00684.x

Turskis, Z.; Gajzler, M.; Dziadosz, A. 2012. Reliability, risk management, and contingency of construction processes and projects, Journal of Civil Engineering and Management 18(2): 290-298.

http://dx.doi.org/10.3846/13923730.2012.672931

Witzany, J.; Cejka, T. 2007. Reliability and failure resistance of the stone bridge structure of Charles bridge during floods, Journal of Civil Engineering and Management 13(3): 227-236.

Yonggang, W.; Yulong, P.; Yangdong, Z. 2008. Vibration-based damage detection with structural modal characteristics, The Baltic Journal of Road and Bridge Engineering 3(1): 21-28. http://dx.doi.org/10.3846/1822-427X.2008.3.21-28

Zwolsky, J.; Bien, J. 2011. Modal analysis of bridge structures by means of forced vibration tests, Journal of Civil Engineering and Management 17(4): 590-599. http://dx.doi.org/10.3846/13923730.2011.632489

Enrique CASTILLO. Received the BS degree in Civil Engineering from the Polytechnic University of Madrid, Madrid, Spain, in 1969, the BS degree in Mathematics from the Complutensis University of Madrid, in 1974, the PhD degree in Civil Engineering from Northwestern University, Chicago, IL, in 1972, and the PhD degree in Civil Engineering from the Polytechnic University of Madrid in 1973. Currently he is a Full Professor in the University of Cantabria, Spain, and a Founding Member of the Royal Academy of Engineering in Spain. His research interests include geotechnics, statistics, Bayesian networks, neuronal networks, artificial intelligence, optimization methods, numerical methods, reliability engineering, structures and sensitivity analysis and its applications.

Jose Antonio LOZANO-GALANT. Assistant Professor at the University of Castilla-La Mancha, Spain. Received his Ph.D degree from the Polytechnic University of Catalonia, BarcelonaTECH in 2013. His research interests include numerical analysis of structures and structural system identification.

Maria NOGAL. Received the BS, MS and PhD degrees in Civil Engineering from the University of Cantabria, Spain, in 2006,2010 and 2011, respectively. Currently she is a Researcher in Trinity College Dublin, Ireland. Her research interests include structural engineering and transport modelling techniques and applications.

Jose TURMO. Associate Professor in the Department of Construction Engineering of the Civil Engineering School at the Universitat Politècnica de Catalunya, BarcelonaTECH, Spain. His research interests include design and construction of concrete structures. 